\title{
Development of Carbon Nanotube TFT for Sheet Electronic Device by Printing Technology
}

\author{
Hiroyuki Endoh \\ Smart Energy Research Laboratories, NEC Corpration \\ 34, Miyukigaoka, Tsukuba, Ibaraki 305-8501, Japan \\ h-endoh@az.jp.nec.com
}

Keywords: carbon nanotube(CNT), thin film transistor(TFT), sheet electronic device

\section{Introduction}

Printed electronics is expected to be low-cost, eco-friendly, and on-demand fabrication technology because it reduces the number of process steps and the amount of waste materials. These are also numbers of applications such as flexible displays, solar cells, RF-ID tags, and sensor sheets. Various materials have been actively proposed as semiconductor materials for printable thin-film transistors to enhance the performance of these applications. We here proposed carbon nanotubes (CNTs) [1] for a printable semiconductor material because they have invaluable advantages over other printable materials[2-4]. The first advantage is chemical and mechanical stability, suitable for durable applications. The second one is high electrical mobility which enables the device operation fast. [5-8]

In this work, we utilized semiconducting carbon nanotubes obtained by electric-field-induced layer formation (ELF) method with nonionic surfactant [9]. In general, ionic dispersants such as sodium dodecyl sulfate are used to disperse CNTs into aqueous solution, but they sometimes cause the device operation unstable. Since ionic species are not used in our separation method, it is favorable for achieving stable device operations. We fabricated $16 \times 16$ CNT-TFT array with a mobility of $3.9 \mathrm{~cm} 2 / \mathrm{Vs}$ with an ability to follow the speed of $1 \mathrm{~m} / \mathrm{s}$.

\section{Method}

\subsection{Structure and characteristics of CNT}

Single-wall CNT has a new form of carbon with unique electrical and mechanical properties. CNT is the material of hollow shape, as shown in Fig.1, is a straw-like structure was rounding a graphene sheet. In general, the diameter of the single-wall CNT is $0.7 \sim 2.0 \mathrm{~nm}$, and the length is $1 \sim 10 \mathrm{um}$. Depending on the folding angle and the diameter, CNTs can be metallic or semiconducting. Simple theory also shows that the band gap of semiconducting CNTs decreases with increasing diameter.

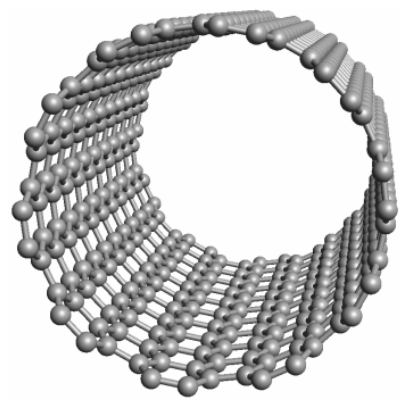

Fig.1. Structure of single-wall CNT.

CNTs have a long one-dimensional structure, the strength of carbon-carbon bond is very strong, and therefore CNTs have excellent stability. TFT using the single-wall CNTs with the very large mobility has been reported[7]. However, it is difficult to fabricate a stable TFTs, because of single-wall CNTs contain $m$-CNTs. By using CNT networks for TFT channel, it is tolerated $m$-CNT contamination, but it is required the purification of the CNTs to achieve high performance of the TFTs.

\subsection{Preparation of carbon nanotubes}

The CNTs with a diameter of $1.3 \mathrm{~nm}$ were used for this study. The CNTs were dispersed into deuterium 
oxide by using nonionic surfactant of polyoxyethylene stearyl ether. Then, the dispersed solution was treated by sonication followed by ultracentrifugation to obtain mono-dispersed CNT solution. After the treatment, metallic and semiconducting CNTs were separated by ELF method repeatedly. Optical absorbance spectra showed that the metallic peaks are suppressed after the treatment. This result indicated that the metallic CNTs were effectively removed from the solutions. The purity of semiconducting CNTs was estimated to be more than $95 \%$ from the spectra(Fig.2 and Fig.3).

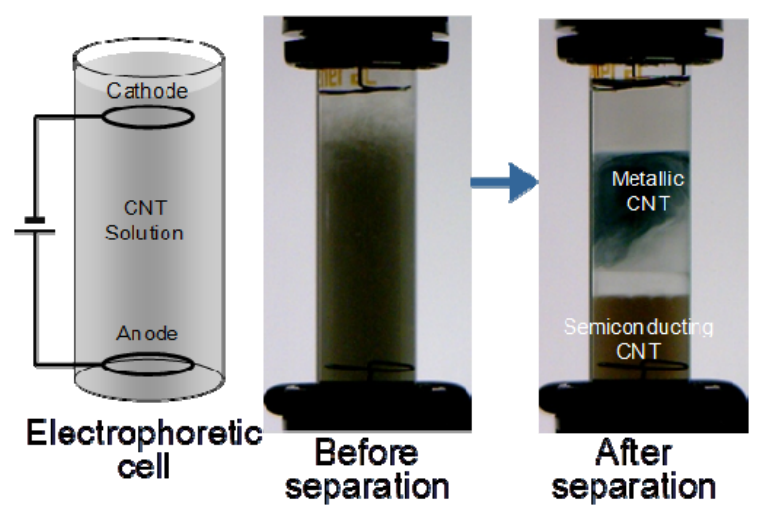

Fig. 2. Schematic of a vertical electrophoretic cell and optical images of CNT solution before and after the voltage application.

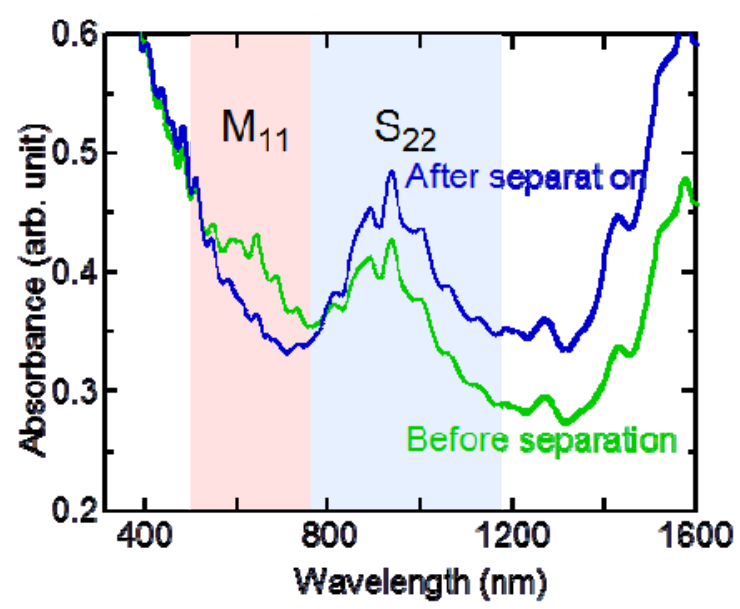

Fig. 3. Absorbance spectra for fraction of CNTs before (green) and after (blue) the separation.

\subsection{Device Fabrication}

CNT-TFT arrays were fabricated on flexible polyimide films. The arrays have source lines and gate lines, each of which connects nearby TFTs. The drain electrodes are connected to pressure-sensitive conductive rubber. The gate electrodes were defined by sputtering gold through a metal mask. The gate insulators were formed by spin coating of polyimide to a thickness of $650 \mathrm{~nm}$, followed by sputter-deposition of gold as source and drain electrodes. Finally, a semiconductor-rich CNT solution was deposited directly on a polyimide film. After the deposition, residual surfactants were removed by heat treatment and wet processes. Atomic-force microscopy revealed that the nanotubes are well dispersed on the polyimide films(Fig.4).

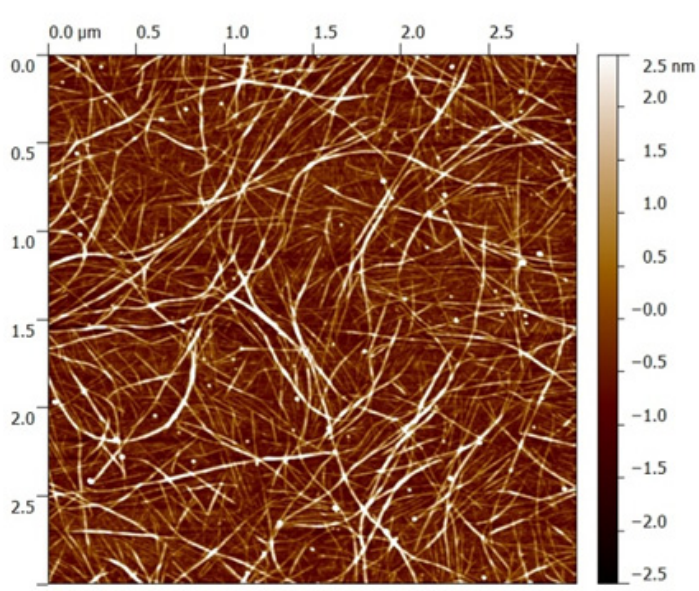

Fig. 4. Atomic force microscopy images of CNT random network on the polyimide film.

\section{Results}

\subsection{Device characteristics}

Drain current is plotted as a function of gate voltage for a typical CNT-TFT in Fig. 5. It is clear from the figure that high drain currents can be controlled by gate voltage, in a similar manner to that of p-type transistors. Due to the utilization of nonionic surfactant to disperse the CNTs, hysteresis in the ID-VGS curve is well suppressed. Statistically, the average mobility is $3.9 \mathrm{~cm}^{2} /(\mathrm{Vs})$ with standard deviation of $55 \%$ with on/off current ratio of $10^{2.4 \pm 0.4}$.(Fig.6-7)

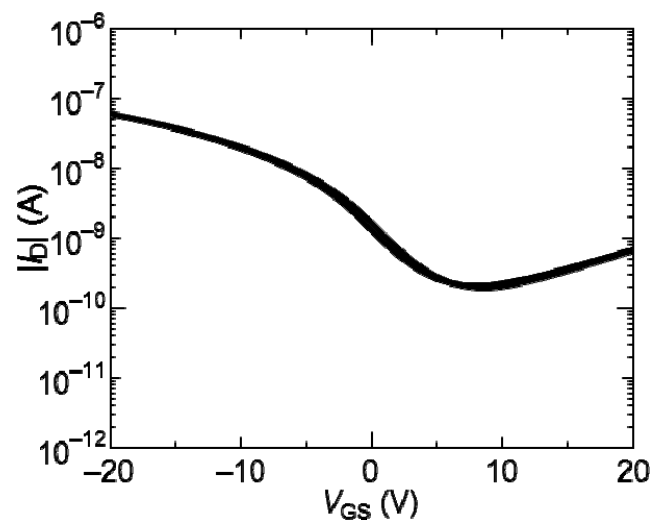

Fig. 5. Drain current $\left(I_{D}\right)$ as a function of gate voltage $\left(\mathrm{V}_{\mathrm{GS}}\right)$ with a drain voltage of $\mathrm{V}_{\mathrm{DS}}=-1 \mathrm{~V}$. 


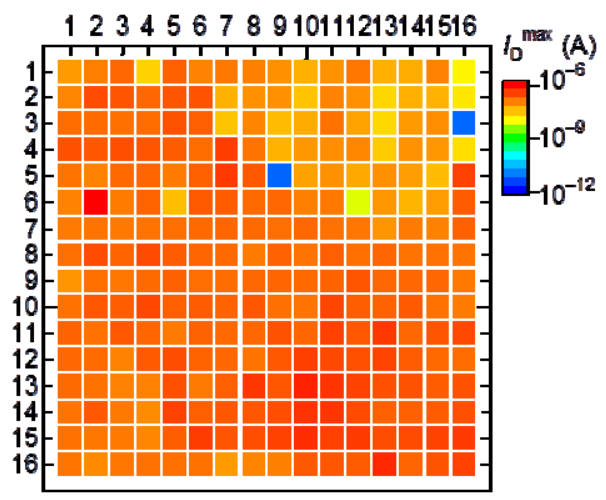

Fig. 6. Color map of ID for $16 \times 16$ CNT-TFT matrix.

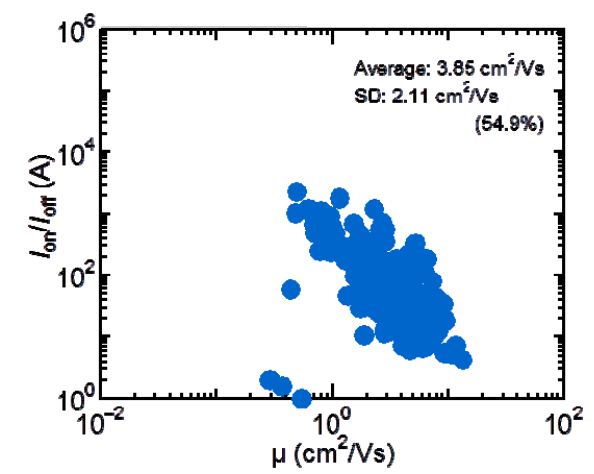

Fig. 7. Dispersion of on/off current ratio and mobility.

We evaluated the functionality of CNT TFT devices as a pressure sensor cell. The pressure-sensing cell was prepared to combine printed CNT TFT, a conductive rubber sheet and a copper-coated film(Fig.8)[10]. The pressure was applied by a needle of a prober system. The drain current changed in response to the applied pressure from $100 \mathrm{nA}$ to $10 \mathrm{pA}$ (Fig.9). These results are promising for pressure sensing applications by using carbon nanotube thin-film transistors.

\subsection{Static and dynamic operation}

An L-shaped object was located on top of a pressure-sensing sheet composed of the fabricated $16 \times 16$ CNT-TFT active-matrix backplane. Clear pattern of the object was obtained by mapping the drain current for the $16 \times 16$ CNT-TFT matrix(Fig.10).

To demonstrate the advantages of pressure-sensing sheets, another TFT sheet was assembled with a pressure-sensing rubber and a counter electrode sheet. As a pressure sensor, this TFT sheet could successfully trace an object moving at a speed of $1 \mathrm{~m} / \mathrm{s}$. This is the first demonstration of tracing a moving object with CNT active-matrix backplanes(Fig.11).

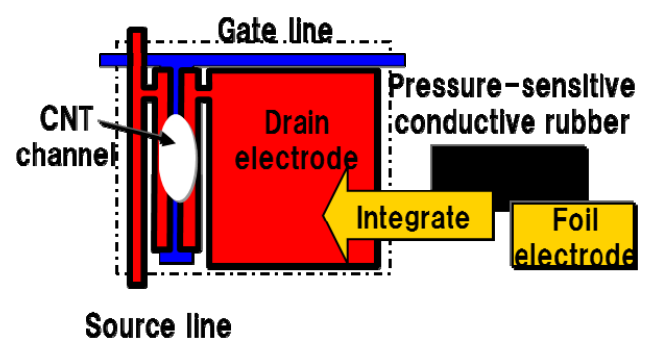

Fig. 8. Layout of CNT-TFT with conductive rubber and counter electrodes.

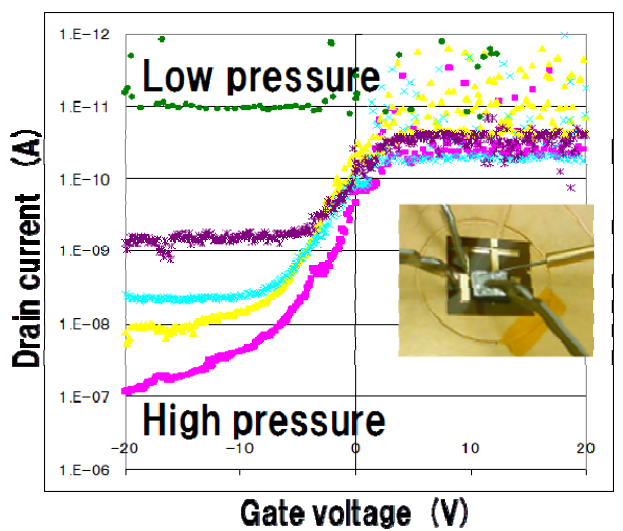

Fig. 9. $\mathrm{I}_{\mathrm{D}}-\mathrm{V}_{\mathrm{GS}}$ profile with a various pressure applied by a probe.

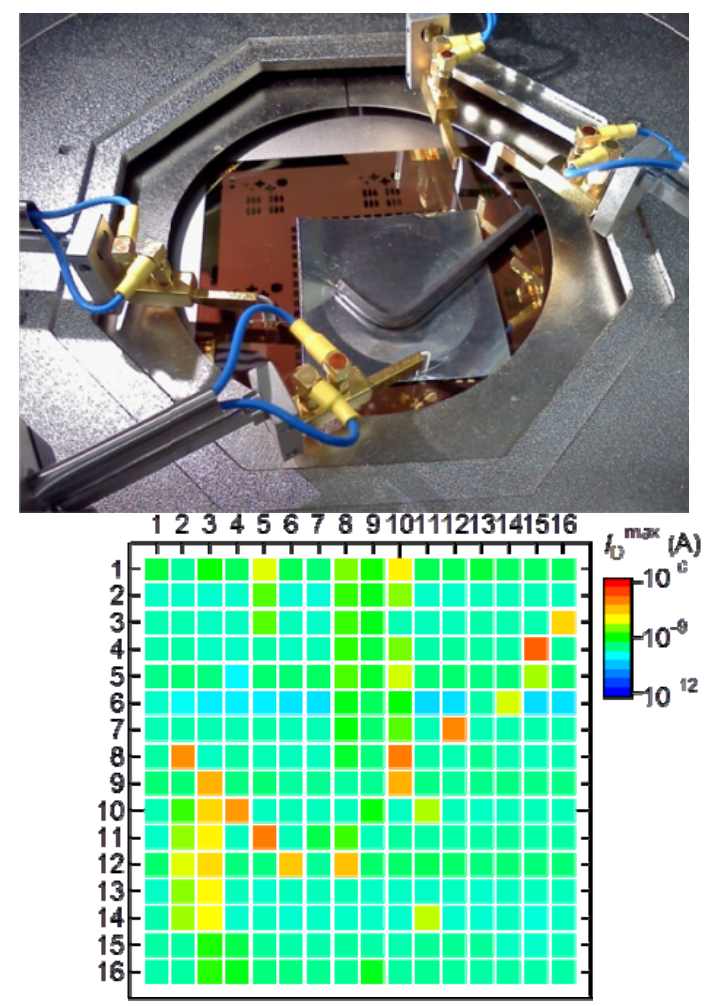

Fig. 10. Photograph of setup with an L-shaped object on pressure sensing sheet and color map of drain current for the $16 \times 16$ CNT-TFT matrix array. 

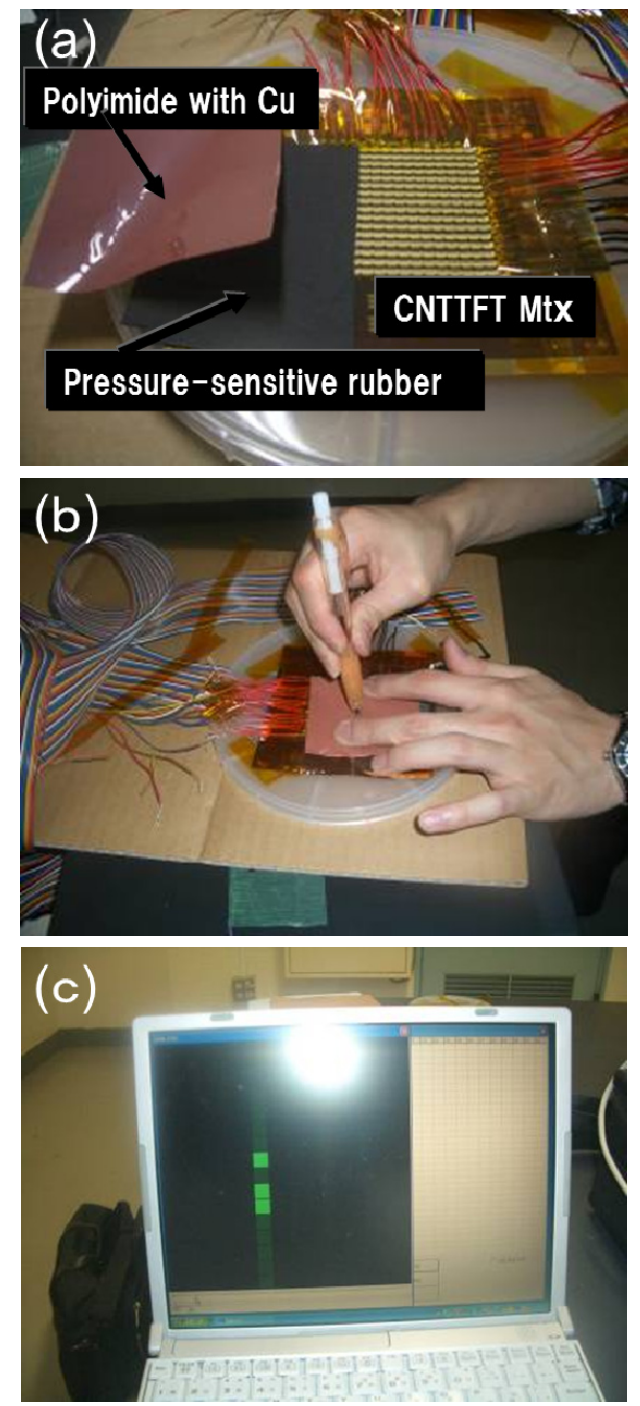

Fig. 11. Photograph of a CNT-TFT active-matrix with a copper-coated polyimide film and a pressure-sensitive conductive rubber(a), application of pressure on the sheet with a stylus pen(b) and Signals from the pressure-sensing sheet displayed on a notebook PC as movement of the pressure point at a speed of about $1 \mathrm{~m} / \mathrm{s}(\mathrm{c})$.

\section{Conclusion}

As a new sheet electronic device, we have discussed the development of pressure-sensing sheet using a CNT-TFT matrix array. Purification of the CNT was successful in the separation of high purity semiconductor CNT in a very simple way. In addition, using the simple printing method, we were able to fabricate high-performance TFT.

This time, we have fabricated a pressure-sensing sheet device using a CNT-TFT matrix array, this is the first demonstration of tracing a moving object with CNT active-matrix backplanes.

\section{Acknowledgement}

The authors thank Dr.Kazuki Ihara for technical advice of CNT purification process. And the authors also thank Mr.Takashi Manako, Ms. Mayumi Kosaka and Mr. Noriyuki Tonouchi for useful discussions.

\section{References}

[1] S. Iijima and T. Ichihashi, Nature, 363 (1993) 603.

[2] M. Ishida and F. Nihey, Appl. Phys. Lett., 92 (2008) 163507.

[3] H. Numata, K. Ihara, T. Saito, H. Endoh and F. Nihey, Appl. Phys. Express, 5 (2012) 055102.

[4] H. Endoh, J.Photopoly.Sci.Technol, 25 (2012), 281.

[5] E. S. Snow, J. P. Novak, P. M. Campbell, and D. Park, Appl. Phys. Lett, 82 (2003) 2145.

[6] E. S. Snow, P. M. Campbell, M. G. Ancona, and J. P. Novak, Appl. Phys. Lett, 86 (2005) 033105.

[7] P. Beecher, P. Servati, A. Rozhin, A. Colli, V. Scardaci, S. Pisana, T. Hasan, A. J. Flewitt, J. Robertson, G. W. Hsieh, F. M. Li, A. Nathan, A. C. Ferrari, and W. I. Milne, J. Appl. Phys., 102 (2007) 043710.

[8] T. Takenobu, N. Miura, S.Y. Lu, H. Okimoto, T. Asano, M. Shiraishi, and Y. Iwasa et al., Appl. Phys. Express, 2 (2009) 025005.

[9] K. Ihara, H. Endoh, T. Saito, and F. Nihey., $J$. Phys. Chem. C, 115 (2011) 22827.

[10] T. Someya, T. Sekitani, S. Iba, Y. Kato, H. Kawaguchi, and T. Sakurai, Proc. Natl. Acad. Sci., 101 (2004) 9966. 